\title{
Long Non-Coding RNA Expression Profiling in Aging Rats with Erectile Dysfunction
}

\author{
Lianjun Pan ${ }^{\mathrm{a} b}$ Jiehua Ma ${ }^{\mathrm{b}}$ Feng Pan ${ }^{\mathrm{b}}$ Dan Zhao Jianping Gao \\ aDepartment of Urology, Jinling Hospital, School of Medicine, Nanjing University, Nanjing, bState Key \\ Laboratory of Reproductive Medicine, Department of Reproductive Health, Nanjing Maternity and \\ Child Health Care Hospital affiliated with Nanjing Medical University, Nanjing, China
}

\section{Key Words}

IncRNAs • Expression profiles • Erectile dysfunction • Gene Ontology • Pathway

\begin{abstract}
Background/Aims: Erectile dysfunction (ED) in aged people remains a topic of interest to andrological physicians. Long non-coding RNAs (IncRNAs), which form the largest group of non-coding RNAs, have been shown to regulate various biological processes. The function of IncRNAs in age-related erectile dysfunction (A-ED) pathogenesis remains poorly understood. Methods: This study aims to assess the differential expression profiles of mRNAs and IncRNAs between A-ED and normal control (NC) samples. Using a second-generation IncRNA microarray, we detected a total of 8,744 IncRNAs and 13,585 coding transcripts. Results: We identified 608 up-regulated and 406 down-regulated IncRNAs in A-ED compared with NC samples, by setting a filter of fold-change $>2$.0. Gene Ontology and pathway analysis revealed that a muscle contraction disorder induced by abnormal ion channels might play a critical role in the pathogenesis of A-ED. Conclusion: Our results show significantly altered expression profiles of IncRNAs and mRNAs between A-ED and NC. This study may provide information for further research on A-ED and may be helpful for finding a new therapeutic target for A-ED.
\end{abstract}

\section{Introduction}

Aging is an important risk factor for erectile dysfunction (ED) [1]. Epidemiologic studies have shown an increasing age-related prevalence and severity of ED [2,3]. Data collected in 29 countries from 27,500 men reveals that approximately $50 \%$ of the men aged $70-80$ years still have sexual intercourse and that $68 \%$ of the men were in favour of using medical treatments

L. Pan and J. Ma contributed equally to this study.

Jianping Gao

KARGER 125
Department of Urology, Jinling Hospital, School of Medicine, Nanjing University, 305rd Zhongshan East Road, Nanjing 210002, (China)

Tel. +86 025-52226162, Fax +86 025-84726922, E-Mail drjianpinggao@163.com 


\section{Cellular Physiology Cell Physiol Biochem 2015;37:1513-1526 \\ \begin{tabular}{ll|l} 
and Biochemistry $10.1159 / 000438519$ & $\begin{array}{l}\text { O 2015 The Author(s). Published by S. Karger AG, Basel } \\
\text { www.karger.com/cpb }\end{array}$ \\
\hline
\end{tabular} \\ Pan et al.: LncRNA Expression Profiling in A-ED Rats}

to help older people enjoy sexual activity [4]. However, the value of medical treatment of ED in the older patient is limited [5]. As the number of older people increases, the needs of older people are expected to become an increasingly relevant health issue. Although age-related ED (A-ED) is attributed largely to endothelial dysfunction and increased oxidative stress in the penis, the molecular basis of this effect remains largely unknown [1]. Therefore, A-ED remains a topic of interest to andrological physicians.

The expression of non-coding RNAs (ncRNAs) appears to be important for mediating erectile function [6]. Long non-coding RNAs (lncRNAs), which are transcripts that are longer than 200 nucleotides (nt) that do not encode proteins, represent a novel class of regulatory molecules [7]. Accumulating evidence indicates that lncRNAs play significant regulatory roles in various biological processes, including genomic imprinting, cell apoptosis, cell differentiation, nuclear trafficking, heat shock response, and genome rearrangement $[8$, 9]. In addition, studies indicate that lncRNAs are associated with a variety of diseases such as cancer, heart disease and neurological disorders [10-12]. Although lncRNAs have been reported to have a wide range of physiological functions, their role in the progression of ED is poorly understood. Several studies have aimed at identifying and characterizing the specific roles of IncRNAs in vascular biology [13]. For example, increased expression of antisense ncRNA transcripts from the INK4 (ANRIL) locus is directly correlated with the severity of atherosclerosis [14]. These observations suggest that lncRNAs may have a functional role in A-ED, which is usually correlated with vascular factors [3].

In this study, we investigated the expression profiles of mRNAs and lncRNAs in A-ED and normal control samples to explore the molecular processes associated with A-ED.

\section{Materials and Methods}

\section{Preparation of tissues}

Samples of the corpora cavernosa in the penis were taken from 20-month old Sprague-Dawley (SD) rats. The Experimental Animal Center of Nanjing Medical University provided the rats, and the Institutional Animal Care and Use committee at Nanjing Medical University approved our study. All of the rats were treated with apomorphine to induced penile erection to evaluate erectile function. Aging rats with ED were screened using a previously published test procedure [15-17]. Then, the experimental rats were divided into two groups, aging rats with normal erectile function as the normal control (NC) group and aging rats with ED (A-ED). Thereafter, histological measurement and intracavernous pressure (ICP) test were used to confirm the groups. Finally, we carefully collected middle penile shafts and corpus cavernosa (CC) from each group. Among the 9 cases for each group, 3 and 6 cases were used for microarray analysis and quantitative real-time polymerase chain reaction (qRT-PCR), respectively. All of the samples were immediately frozen in liquid nitrogen and then stored at $-80^{\circ} \mathrm{C}$ prior to analysis.

\section{RNA extraction and quality control}

Total RNA derived from rat penile tissue was obtained using TRIzol Reagent (Invitrogen, Carlsbad, CA, USA) following a standard extraction protocol and then purified using an RNeasy Mini Kit (Qiagen, Hilden, Germany) according to the manufacturer's instructions. The quantification and quality of RNA samples were assessed on a NanoDrop ND-1000 Spectrophotometer (Thermo Scientific) and an Agilent 2100 Bioanalyzer (Agilent Technologies), respectively. The OD260/280 absorbance ratios were between 1.8 and 2 for all samples, and RNA integrity was assessed using standard denaturing agarose gel electrophoresis.

\section{Microarray analysis}

For microarray analysis, the Agilent array platform was employed. Sample preparation and microarray hybridization were performed based on the manufacturer's standard protocols. Briefly, total RNA from each sample was amplified and transcribed into fluorescent cRNA using the Agilent's Quick Amp Labelling protocol (version 5.7, Agilent Technologies). The labelled cRNAs were hybridized onto the rat LncRNA Array v2.0 (4 x 44K, Arraystar). The arrays were scanned on an Agilent Scanner G2505C after having washed the slides. The analysis of acquired array images was performed using Agilent Feature Extraction software 


\section{Cellular Physiology Cell Physiol Biochem 2015;37:1513-1526 \begin{tabular}{ll|l} 
and Biochemistry & $\begin{array}{l}\text { DOI: 10.1159/000438519 } \\
\text { Published online: October 30, } 2015\end{array}$ & $\begin{array}{l}\text { C 2015 The Author(s). Published by S. Karger AG, Basel } \\
\text { www.karger.com/cpb }\end{array}$ \\
\hline
\end{tabular} Pan et al.: LncRNA Expression Profiling in A-ED Rats}

(version 11.0.1.1). The GeneSpring GX v11.5.1 software package (Agilent Technologies) was used to carry out quantile normalization and subsequent data processing. After quantile normalization of the raw data, IncRNAs and mRNAs with present or marginal flags in at least 1 out of 2 samples ("All Targets Value") were chosen for further data analysis.

Quantitative real-time PCR ( $q R T-P C R)$

To validate the microarray data, we investigated the expression patterns of 12 selected lncRNAs that were identified as differentially expressed in two groups using qRT-PCR. Primers were designed online using Primer3 (http://sourceforge.net/projects/primer3/) and evaluated using Basic Local Alignment Search Tool (BLAST) at NCBI to ensure unique amplification products. All of the primer sequences are shown in Table 1. The SYBR green method was used to perform qRT-PCR. The reactions were incubated in a 96-well optical plate at $95^{\circ} \mathrm{C}$ for $10 \mathrm{~min}$, followed by $40 \times$ PCR cycles of $95^{\circ} \mathrm{C}$ for $15 \mathrm{~s}$ and $60^{\circ} \mathrm{C}$ for $1 \mathrm{~min}$. Gene expression levels were quantified based on the cycle threshold (Ct) values and values are expressed as $2-\Delta \Delta \mathrm{CT}$. The relative levels of lncRNAs were normalized to glyceraldehyde 3-phosphate dehydrogenase (GAPDH).

Gene Ontology (GO) and pathway analysis

In this study, GO and pathway analyses were performed to determine the gene and gene product attributes in GO terms or biological pathways. Fisher's exact test was used to determine whether there is more overlap between the differentially expressed (DE) list of genes and the GO annotation list than would be expected by chance. The GO categories are derived from the Gene Ontology database (http://www. geneontology.org), which comprise three structured networks of defined terms that describe gene product attributes. The ontology covers three domains, biological process, cellular component and molecular function. The lower the p-value, the more significant the GO terms enrichment in DE genes (p-value $<0.05$ is recommended). Pathway analysis is a functional analysis that maps genes to KEGG (Kyoto Encyclopedia of Genes and Genomes, http://www.genome.jp/kegg) pathways. Khatri et al. reported that pathway analysis has become the first choice for learning the underlying biology of differentially expressed genes and proteins [18]. The p-value (Fisher's p-value, Hypergeometric p-value or EASE-score) denotes the significance of the pathway correlated to the conditions. The lower the $\mathrm{p}$-value, the more significant is the pathway.

\section{Statistical analysis}

The SPSS 17.0 software package (SPSS, Chicago, IL, USA) was used to analyse the data. Differential expression levels of lncRNAs were compared by independent-samples t-test between two groups. Fisher's exact test was used for GO and pathway analysis. Statistical significance was defined as $\mathrm{P}<0.05$.

Table 1. The primer sequences used in this study

\begin{tabular}{lllc}
\hline Target ID & Forward Primer & Reverse Primer & Product Length (bp) \\
\hline GAPDH & F:5'GGAAAGCTGTGGCGTGAT3' & R:5'AAGGTGGAAGAATGGGAGTT3' & 308 \\
MRAK080737 & F:5'CCAGTGACAGGGGAAAGCA3' & R:5'GGAGATGGGCAGGATGATG3' & 95 \\
MRUC008SUT & F:5'CCATACACGCATGACTCAATCA3' & R:5'CAGGTGCCTCCAGTCTTCTT3' & 130 \\
AB049626 & F:5'AGGAAAGGGCTGACTGGAA3' & R:5'CTTGGGATGTCTGTCTTTGAG3' & 121 \\
MRNR_026574 & F:5'GCACGCCTTCTCTGTCTCT3' & R:5'GCATTCGCTGATAGTCCTTCC3' & 296 \\
MRAK161159 & F:5'TCTCCTGGTAATGCTGTTCCT3' & R:5'GCAGTGGCTTCTCAATGTCTT3' & 66 \\
MRAK040414 & F:5'GGTGCGGTCTCTTGAGGTGA3' & R:5'GGTCTCACAGGTTCCATTCAGT3' & 155 \\
MRAK153573 & F:5'GTCGGCTTGGAATCACAGTAG3' & R:5'AAGAGCATTGATGTAGGCAGTG3' & 252 \\
MRAK078930 & F:5'AAACCCAGAATACCCAGTGC3' & R:5'GGAACCCAGCAGACCAACTA3' & 134 \\
BC167026 & F:5'TTGTTCTCAGTTTCTCGGTTTA3' & R:5'TGGTAGAGGTCCAGAAGGTT3' & 177 \\
MRAK142111 & F:5'GCAAGCAGATGGTGTTGAGTAA3' & R:5'GGCAGGATGAGGCATTATTGTT3' & 70 \\
BC082040 & F:5'TGCTGTCAGGCTGCTCAT3' & R:5'GCTTGCTCCGATTGTCCATT3' & 171 \\
MRAK084895 & F:5'ACTCGCCCAGTTTTGCAAG3' & R:5'TTTAAATGATGACATTCCCGAAG3' & 185 \\
\hline
\end{tabular}




\section{Cellular Physiology Cell Physiol Biochem 2015;37:1513-1526 \begin{tabular}{ll|l} 
DOI: 10.1159/000438519 & $\begin{array}{l}\text { O } 2015 \text { The Author(s). Published by S. Karger AG, Basel } \\
\text { www.karger.com/cpb }\end{array}$ \\
\cline { 2 - 3 }
\end{tabular} Pan et al.: LncRNA Expression Profiling in A-ED Rats}

\section{Results}

Overview of the microarray data

Global profiling of rat lncRNAs and protein-coding transcripts was performed using the rat LncRNA Array v2.0 (4 x 44K, Arraystar). The microarray data showed that a total of 8,744 lncRNAs and 13,585 coding transcripts were detected. The lncRNAs come from an authoritative database such as UCR, RefSeq_XR and misc_lncRNA (Fig. 1A) [19-22]. A scatterplot was used for assessing the expression variation (or reproducibility) between chips. The green lines are fold change lines (The default fold change value given is 2). The lncRNAs (or mRNAs) above the top green line and below the bottom green line indicates more than 2 fold change of lncRNAs (or mRNAs) between the two samples (or samples) (Fig. 1B, C). Based on these data, the IncRNA expression levels between the A-ED group and the NC group were compared. By applying a filter of fold-change $>2.0$, we identified 608 up-regulated and 406 down-regulated lncRNAs. The list of the top 50 up- and down-regulated lncRNAs and mRNAs identified by microarray analysis is shown in Tables $2-5$.

Validation of microarray results by $q R T-P C R$

To validate the microarray results, we selected 12 differentially expressed IncRNAs according to their associated mRNAs [23, 24]. 7 up-regulated lncRNAs and 5 downregulated IncRNAs (MRAK078930, were analysed using qRT-PCR in 20 pairs of A-ED and matched NC tissues. qRT-PCR results showed that MRAK080737, MRUC008SUT, AB049626, MRNR_026574, MRAK161159, MRAK040414, MRAK153573 increased, whereas MRAK078930, BC167026, MRAK142111, BC082040 and MRAK084895 decreased in A-ED (all $\mathrm{P}<0.05$, Fig. 2). According to the qRT-PCR analysis, we further found that the most upregulated IncRNAs were MRAK080737 and MRuc008sut, whereas the most down-regulated lncRNAs were MRAK078930 and BC167026. These results indicate that the expression trends of the selected lncRNAs are consistent with the microarray data, which demonstrates

Fig. 1. Profile of the microarray data. A total of 8,744 lncRNAs and 13,585 coding transcripts were detected. (A) The pie chart shows the number of IncRNAs from each authoritative database. (B) The IncRNAs above the top green line and below the bottom green line show a more than 2.0-fold change in lncRNA expression between the A-ED and NC samples. (C) The mRNAs above the top green line and below the bottom green line show a more than 2.0-fold change of mRNA expression between the A-ED and NC samples.

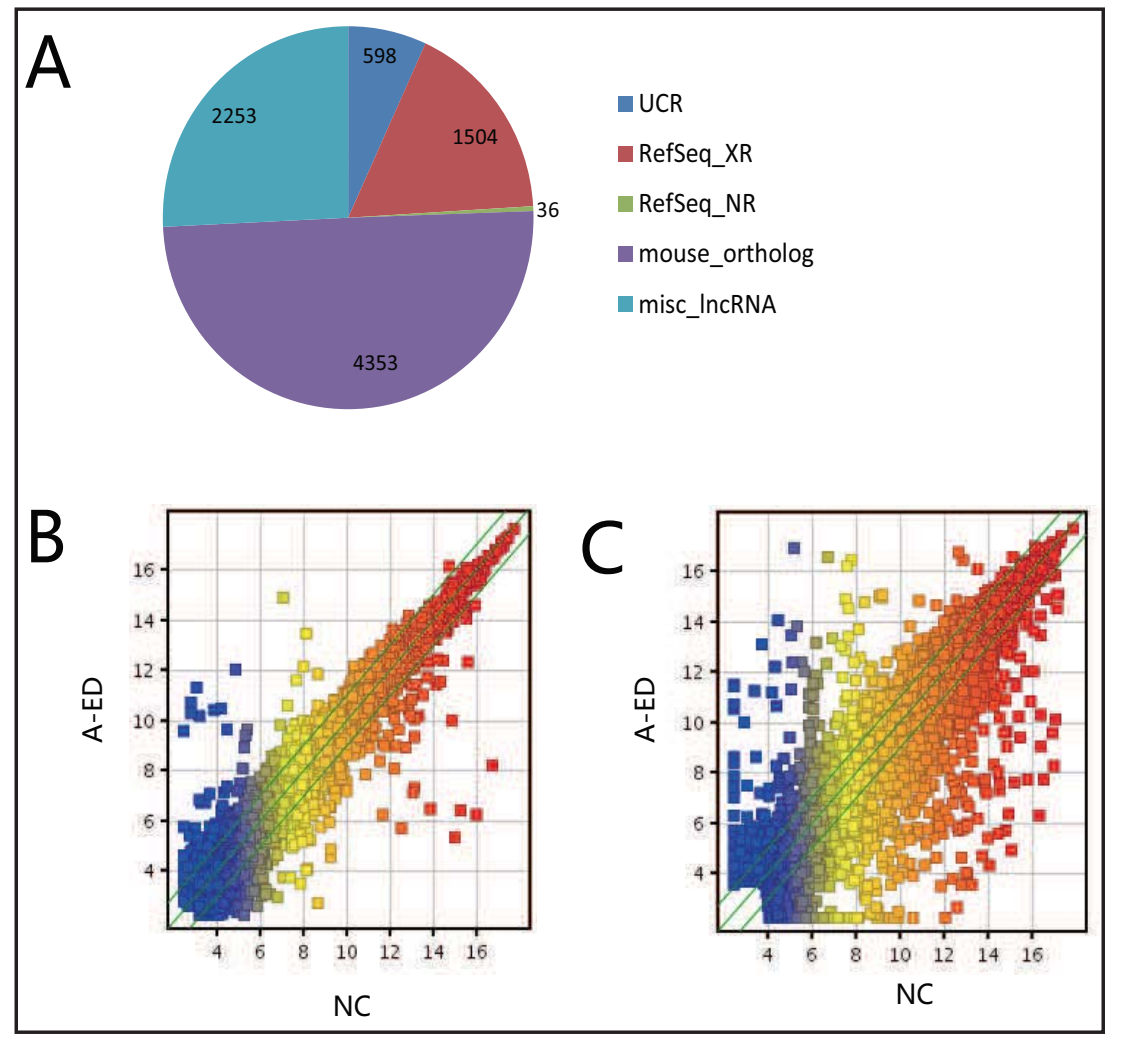



Cellular Physiology Cell Physiol Biochem 2015;37:1513-1526 \begin{tabular}{c|c|c|c|}
\cline { 2 - 3 } DOI: 10.1159/000438519 & (c) 2015 The Author(s). Published by S. Karger AG, Basel
\end{tabular} and Biochemistry Published online: October 30, 2015 www.karger.com/cpb

Pan et al.: LncRNA Expression Profiling in A-ED Rats

Table 2. Top 50 up-regulated lncRNAs in the A-ED group compared with the NC group and filtered by a fold-change $>3.0$

\begin{tabular}{|c|c|c|c|c|c|c|}
\hline SeqID & $\begin{array}{l}\text { Fold change } \\
(A-E D / N C)\end{array}$ & Chromosome & Strand & Relationship & Associated_gene_acc & $\begin{array}{c}\text { Associated_gene_ } \\
\text { name }\end{array}$ \\
\hline MRAK033351 & 336.84 & chr17 & + & intergenic & & \\
\hline L38717 & 255.60 & chr3 & - & intergenic & & \\
\hline K02111 & 244.73 & chr10 & + & sense_exon_overlap & NM_001100485 & \\
\hline U89530 & 192.16 & chr3 & - & intergenic & & \\
\hline AF059344 & 157.25 & chr3 & - & intergenic & & \\
\hline U89531 & 151.39 & chr3 & - & intergenic & & \\
\hline MRAK020463 & 141.30 & chr3 & - & others & & \\
\hline AF525411 & 103.59 & chr3 & - & intergenic & & \\
\hline AF525412 & 86.58 & chr3 & - & intergenic & & \\
\hline X90475 & 43.11 & chr7 & - & sense_exon_overlap & NM_001100758 & \\
\hline EU056364 & 37.95 & chr18 & + & sense_exon_overlap & NM_012920 & Camk2a \\
\hline AF130879 & 20.03 & chr1 & - & others & & \\
\hline AY167411 & 18.81 & $\operatorname{chr} 10$ & - & others & & \\
\hline XR_007519 & 18.51 & chr5 & - & intergenic & & \\
\hline MRAK080737 & 16.78 & chr4 & - & bidirection & NM_022522 & Casp2 \\
\hline AY429596 & 14.27 & chr3 & - & intergenic & & \\
\hline AY177417 & 13.87 & $\operatorname{chr} 16$ & - & sense_exon_overlap & NM_001169141 & \\
\hline XR_009380 & 11.68 & chrUn & - & others & & \\
\hline MRuc008ytr & 11.50 & chrUn & - & others & & \\
\hline MRAK081751 & 10.81 & chr5 & + & sense_intron_overlap & NM_001106630 & Jph1 \\
\hline AY177416 & 10.75 & chr9 & + & intergenic & & \\
\hline MRuc008lvx & 10.39 & chr3 & + & sense_exon_overlap & NM_001145840 & Ganc \\
\hline MRuc008lvx & 10.39 & chr3 & + & sense_exon_overlap & NM_017117 & Capn3 \\
\hline MRNR_002452 & 10.02 & chr1 & - & others & & \\
\hline MRAK033229 & 8.12 & chr4 & + & others & & \\
\hline XR_008345 & 7.96 & chr10 & - & intergenic & & \\
\hline MRuc008sut & 7.88 & chr5 & + & sense_exon_overlap & NM_031628 & $\mathrm{Nr} 4 \mathrm{a} 3$ \\
\hline MRAK134248 & 7.67 & chr1 & + & sense_exon_overlap & NM_030585 & Rabep2 \\
\hline XR_009626 & 7.16 & chr4_random & + & intergenic & & \\
\hline MRAK044354 & 6.84 & chr3 & - & others & & \\
\hline MRuc008xkb & 5.85 & chr14 & + & others & & \\
\hline uc.78+ & 5.62 & chr3 & + & $\begin{array}{l}\text { antisense_intron_overla } \\
\mathrm{p}\end{array}$ & NM_001033701 & Zeb2 \\
\hline XR_006950 & 5.12 & chr4 & - & intergenic & & \\
\hline MRAK050904 & 5.11 & chr17 & + & others & & \\
\hline uc. $346+$ & 5.09 & chr7 & - & intergenic & & \\
\hline uc.466- & 5.06 & $\operatorname{chrX}$ & + & $\begin{array}{l}\text { antisense_intron_overla } \\
\mathrm{p}\end{array}$ & NM_053479 & \\
\hline uc. $432-$ & 5.04 & $\operatorname{chr} 18$ & - & intergenic & & \\
\hline MRAK014008 & 5.01 & chr2 & - & sense_exon_overlap & NM_031130 & Nr2f1 \\
\hline MRuc008czh & 4.85 & chr9 & + & sense_intron_overlap & NM_001012129 & Satb1 \\
\hline uc.253- & 4.78 & chr5 & - & intergenic & & \\
\hline uc. $162+$ & 4.76 & chr2 & - & intergenic & & \\
\hline MRAK032315 & 4.69 & $\operatorname{chrX}$ & + & intergenic & & \\
\hline BC063168 & 4.57 & chr4 & - & sense_exon_overlap & NM_017102 & Slc2a3 \\
\hline MRAK021118 & 4.50 & $\operatorname{chr} 9$ & - & others & & \\
\hline AY390391 & 4.43 & $\operatorname{chr} 17$ & + & intergenic & & \\
\hline AF112256 & 4.37 & chr1 & $\cdot$ & intergenic & & \\
\hline XR_009508 & 4.34 & chr3 & + & intergenic & & \\
\hline XR_005466 & 4.26 & $\operatorname{chr} 10$ & - & intergenic & & \\
\hline DQ402472 & 4.26 & chr6 & - & intergenic & & \\
\hline XR_006612 & 4.24 & chr4 & + & others & & \\
\hline
\end{tabular}

that the microarray data were stable and reliable. The fold-change of the selected lncRNAs, as validated by the microarray analysis and RT-PCR, are depicted in Table 6 . Thus, our results show that a set of IncRNAs are consistently differentially expressed in A-ED and NC and might be related to A-ED pathogenesis.

GO and pathway analysis

GO term enrichment analysis was performed to evaluate the associated coding gene of the dysregulated IncRNAs. As shown in Fig. 3A, the top $10 \mathrm{GO}$ terms for the up-regulated genes in our study included developmental process, multicellular organismal development, muscle system process, system development, muscle contraction, anatomical structure development, muscle structure development, cell differentiation, ion transport and organ development. Whereas, the top $10 \mathrm{GO}$ terms on the down-regulated IncRNAs involved were 

Cellular Physiology Cell Physiol Biochem 2015;37:1513-1526

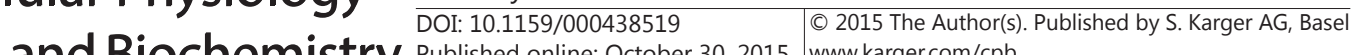 and Biochemistry Published online: October 30, 2015 www.karger.com/cpb

Pan et al.: LncRNA Expression Profiling in A-ED Rats

Table 3. Top 50 down-regulated IncRNAs in the A-ED group compared with the NC group and filtered by a fold-change $>3.0$

\begin{tabular}{|c|c|c|c|c|c|c|}
\hline SeqID & $\begin{array}{l}\text { Fold change } \\
(A-E D / N C)\end{array}$ & $\begin{array}{l}\text { Chromo } \\
\text { some }\end{array}$ & Strand & Relationship & Associated_gene_acc & $\begin{array}{c}\text { Associated_gene } \\
\text { name }\end{array}$ \\
\hline XR_008965 & -822.06 & chr4 & + & others & & \\
\hline XR_008714 & -753.09 & $\operatorname{chr} 3$ & - & intergenic & & \\
\hline BC086588 & -424.34 & chr2 & - & sense_intron_overlap & NM_001109500 & Lce1 m \\
\hline BC166816 & -339.13 & chr1 & + & sense_exon_overlap & NM_001173357 & \\
\hline MRAK009853 & -160.78 & chr2 & - & intergenic & & \\
\hline uc. $150+$ & -105.39 & chr17 & + & intergenic & & \\
\hline MRAK004471 & -58.33 & chr17 & + & intergenic & & \\
\hline XR_005499 & -56.41 & chr3 & - & intergenic & & \\
\hline MRAK010032 & -51.39 & chr4 & + & others & & \\
\hline XR_007003 & -38.79 & chrUn & - & others & & \\
\hline MRAK010042 & -26.44 & chr18 & + & sense_exon_overlap & NM_001170606 & \\
\hline BC161881 & -23.49 & chr7 & + & others & & \\
\hline BC092202 & -20.21 & $\operatorname{chr} 10$ & - & others & & \\
\hline AY044435 & -19.41 & chr7 & + & others & & \\
\hline BC087706 & -15.98 & chr2 & - & intergenic & & \\
\hline XR_008731 & -15.72 & chr1 & + & others & & \\
\hline BC129114 & -15.67 & chr1 & + & intergenic & & \\
\hline XR_008801 & -13.37 & chr8 & - & intergenic & & \\
\hline MRAK011597 & -13.08 & $\operatorname{chrX}$ & + & others & & \\
\hline M21759 & -12.86 & chr2 & + & intergenic & & \\
\hline XR_008601 & -11.57 & $\operatorname{chr} 9$ & + & intergenic & & \\
\hline MRAK028845 & -9.56 & $\operatorname{chr} 10$ & + & antisense_exon_overlap & NM_001008759 & Krt1-5 \\
\hline MRAK076299 & -9.49 & chrUn & + & intergenic & & \\
\hline MRuc007pas & -9.10 & chr6 & + & intergenic & & \\
\hline ВС092641 & -8.92 & chr3 & - & antisense_exon_overlap & NM_001100531 & Dlx1 \\
\hline XR_009334 & -8.80 & chr1 & + & others & & \\
\hline BC098071 & -8.76 & $\operatorname{chr} 17$ & - & others & & \\
\hline BC160888 & -8.59 & chr5 & + & sense_exon_overlap & NM_001135780 & RGD1304693 \\
\hline AY321319 & -8.42 & chr9 & - & others & & \\
\hline MRAK013532 & -8.07 & chr6 & + & intergenic & & \\
\hline MRAK078930 & -8.03 & chr1 & - & sense_exon_overlap & NM_013159 & Ide \\
\hline XR_009344 & -7.67 & chr7 & + & others & & \\
\hline BC091361 & -7.54 & $\operatorname{chr} 10$ & + & others & & \\
\hline BC167069 & -7.50 & chr3 & - & bidirection & NM_001107762 & Nusap1 \\
\hline BC167026 & -7.49 & chr15 & + & sense_exon_overlap & NM_001106028 & Cdkn3 \\
\hline MRAK048947 & -7.45 & chr1 & + & others & & \\
\hline uc. $176+$ & -7.31 & $\operatorname{chr} 10$ & - & sense_intron_overlap & NM_020088 & Odz2 \\
\hline MRAK142484 & -7.01 & chr18 & - & sense_exon_overlap & NM_031826 & $\mathrm{Fbn} 2$ \\
\hline BC107469 & -6.45 & $\operatorname{chr} 16$ & + & others & & \\
\hline AJ224880 & -6.30 & chr9 & - & sense_exon_overlap & NM_053488 & \\
\hline MRAK009873 & -6.25 & chr8 & + & others & & \\
\hline MRAK012306 & -6.23 & chr6 & - & others & & \\
\hline AB011529 & -6.21 & chr2 & - & others & & \\
\hline AF370442 & -6.19 & $\operatorname{chr} 13$ & - & sense_exon_overlap & NM_001100827 & \\
\hline BC158675 & -6.10 & chr6 & + & intergenic & & \\
\hline MRuc008qeu & -6.05 & chr2 & - & others & & \\
\hline MRAK132609 & -5.89 & chr2 & + & intergenic & & \\
\hline MRuc008fxu & -5.66 & chr1 & + & others & & \\
\hline MRuc009gus & -5.63 & chr1 & - & others & & \\
\hline BC166461 & -5.58 & $\operatorname{chr} 15$ & - & sense_exon_overlap & NM_001108379 & RGD1307201 \\
\hline
\end{tabular}

cell proliferation, epidermis development, organ development, regulation of peptidase activity, developmental process, tissue development, regulation of biological quality, anatomical structure development, negative regulation of molecular function, and regulation of cell proliferation (Fig. 3C).

Pathway analysis indicated that the top 10 pathways corresponding to the associated coding gene of up-regulated lncRNAs are involved in the following pathways: gastric acid 

Cellular Physiology Cell Physiol Biochem 2015;37:1513-1526

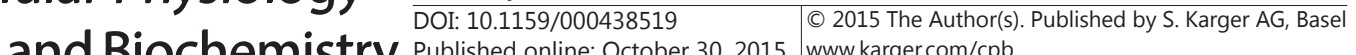 and Biochemistry Published online: October 30, 2015 www.karger.com/cpb
Pan et al.: LncRNA Expression Profiling in A-ED Rats

Table 4. The top 50 up-regulated mRNAs in the A-ED group compared with the NC group and filtered by a fold-change $>3.0$

\begin{tabular}{|c|c|c|c|c|c|}
\hline Gene Symbol & Fold change $(\mathrm{A}-\mathrm{ED} / \mathrm{NC})$ & Chromosome & Strand & txStart & txEnd \\
\hline Myh4 & 3733.56 & $\operatorname{chr} 10$ & + & 53932089 & 53955029 \\
\hline Actn3 & 1019.18 & chr1 & - & 207475568 & 207492267 \\
\hline Myh1 & 837.57 & $\operatorname{chr} 10$ & + & 53894387 & 53917948 \\
\hline Mylpf & 711.12 & $\operatorname{chr} 1$ & + & 186471804 & 186474560 \\
\hline Sypl2 & 570.27 & $\operatorname{chr} 2$ & - & 203777721 & 203792453 \\
\hline Apobec2 & 562.42 & chr9 & + & 7947499 & 7961013 \\
\hline Myl1 & 421.66 & chr9 & - & 65717097 & 65727679 \\
\hline Myl1 & 410.70 & chr9 & - & 65717097 & 65737576 \\
\hline Pvalb & 375.64 & $\operatorname{chr} 7$ & - & 116115033 & 116127508 \\
\hline Myl3 & 363.98 & chr8 & + & 115140704 & 115146827 \\
\hline Myf6 & 343.88 & chr7 & - & 46199971 & 46201867 \\
\hline Art5 & 301.77 & chr1 & - & 159576989 & 159586949 \\
\hline Art1 & 253.86 & $\operatorname{chr} 1$ & + & 159586245 & 159590797 \\
\hline Trdn & 250.63 & chr1 & - & 24514751 & 24977515 \\
\hline Myoz1 & 177.60 & $\operatorname{chr} 15$ & + & 3869804 & 3877019 \\
\hline Ampd1 & 169.07 & chr2 & + & 198308511 & 198331019 \\
\hline Lmod2 & 148.49 & chr4 & + & 410044 & 417739 \\
\hline Cmya3 & 135.65 & chr3 & + & 49414868 & 49502135 \\
\hline Mybpc2 & 135.57 & chr1 & - & 94979307 & 95002804 \\
\hline Myot & 127.82 & $\operatorname{chr} 18$ & + & 38003999 & 38023531 \\
\hline Tnni2 & 122.91 & $\operatorname{chr} 1$ & + & 202687629 & 202690172 \\
\hline Cox6a 2 & 91.67 & chr1 & - & 187464578 & 187465325 \\
\hline Stac3 & 90.16 & chr7 & + & 67478505 & 67485909 \\
\hline $\mathrm{Ckm}$ & 89.26 & $\operatorname{chr} 1$ & + & 78762201 & 78772681 \\
\hline Mypn & 83.91 & chr20 & - & 25457132 & 25531818 \\
\hline Kenj11 & 83.04 & $\operatorname{chr} 1$ & - & 96614959 & 96617993 \\
\hline LOC680319 & 79.70 & $\operatorname{chrX}$ & + & 123537651 & 123539744 \\
\hline Tpm1 & 66.74 & chr8 & - & 71336842 & 71358063 \\
\hline Myod1 & 66.73 & $\operatorname{chr} 1$ & + & 96910189 & 96912900 \\
\hline Hspb3 & 66.68 & chr2 & - & 45078614 & 45079329 \\
\hline Tpm1 & 63.52 & chr8 & - & 71336842 & 71352726 \\
\hline Ankrd23 & 59.19 & chr9 & - & 35472714 & 35479314 \\
\hline Tpm1 & 57.02 & chr8 & - & 71336842 & 71352726 \\
\hline Tnnt3 & 55.86 & chr1 & + & 202748062 & 202761965 \\
\hline $\mathrm{Mb}$ & 54.79 & chr7 & - & 115087557 & 115094789 \\
\hline Cox8h & 53.36 & chr1 & - & 201057313 & 201058774 \\
\hline Casq1 & 50.21 & $\operatorname{chr} 13$ & - & 88200505 & 88210418 \\
\hline Klhl31 & 48.72 & chr8 & + & 82609179 & 82633422 \\
\hline Nrap & 47.33 & chr1 & - & 262591453 & 262672255 \\
\hline $\mathrm{Hfe} 2$ & 42.52 & chr2 & + & 191329643 & 191333525 \\
\hline Myh2 & 40.87 & chr10 & + & 53864776 & 53891711 \\
\hline Kbtbd5 & 40.70 & chr8 & - & 127153471 & 127159307 \\
\hline Asb10 & 39.41 & chr4 & + & 6010912 & 6019444 \\
\hline Ckmt2 & 35.43 & chr2 & - & 22084949 & 22106014 \\
\hline Arx & 34.93 & $\operatorname{chrX}$ & + & 80631374 & 80643987 \\
\hline Aqp 4 & 29.58 & chr18 & - & 6626312 & 6641850 \\
\hline Trim54 & 26.36 & chr6 & - & 25218007 & 25237575 \\
\hline Aqp 4 & 24.13 & chr18 & - & 6626312 & 6642766 \\
\hline Csrp3 & 24.09 & chr1 & - & 98601680 & 98611747 \\
\hline RGD1310090 & 24.00 & chr16 & - & 64952302 & 64959604 \\
\hline
\end{tabular}

secretion, calcium signalling, pancreatic secretion, dilated cardiomyopathy, arrhythmogenic right ventricular cardiomyopathy (ARVC), insulin secretion, vascular smooth muscle contraction, hypertrophic cardiomyopathy (HCM), neuroactive ligand-receptor interaction, and fat digestion and absorption (Fig. 3B). The top 10 pathway corresponding to the associated coding gene of down-regulated lncRNAs are involved in protein digestion and 

Cellular Physiology Cell Physiol Biochem 2015;37:1513-1526 \begin{tabular}{ll|l} 
DOI: 10.1159/000438519 & 2015 The Author(s). Published by S. Karger AG, Basel \\
\hline and Biochemistry
\end{tabular} and Biochemistry Published online: October 30, 2015 www.karger.com/cpb
Pan et al.: LncRNA Expression Profiling in A-ED Rats

Table 5. The top 50 down-regulated mRNAs in the A-ED group compared with the NC group and filtered by a fold-change $>3.0$

\begin{tabular}{|c|c|c|c|c|c|}
\hline Gene Symbol & Fold change (A-ED/ NC) & Chromosome & Strand & txStart & txEnd \\
\hline Vcsa1 & -1018.00 & chr14 & $\cdot$ & 21378052 & 21382729 \\
\hline $\mathrm{Stfa} 3$ & -949.71 & chr11 & - & 66030826 & 66035282 \\
\hline Il1f9 & -916.86 & chr3 & + & 2432284 & 2438436 \\
\hline Serpinb12 & -810.47 & $\operatorname{chr} 13$ & + & 13106079 & 13137360 \\
\hline Serpinb13 & -803.10 & $\operatorname{chr} 13$ & + & 13175745 & 13209693 \\
\hline Kprp & -761.34 & chr2 & - & 185839395 & 185843694 \\
\hline Cldn17 & -560.59 & chr11 & - & 28370003 & 28371180 \\
\hline Defb4 & -521.12 & $\operatorname{chr} 16$ & - & 75442004 & 75445196 \\
\hline Fgfbp1 & -515.95 & chr14 & + & 72242270 & 72243353 \\
\hline Aqp5 & -485.23 & chr7 & + & 138337730 & 138341257 \\
\hline Muc10 & -461.11 & $\operatorname{chr} 14$ & - & 21294394 & 21294762 \\
\hline Csta & -423.85 & $\operatorname{chr} 11$ & + & 66456247 & 66467253 \\
\hline RGD1562234 & -422.57 & chr2 & + & 183499182 & 183502085 \\
\hline Klk12 & -357.36 & chr1 & + & 94198529 & 94203133 \\
\hline Slc15a1 & -353.79 & $\operatorname{chr} 15$ & - & 106550095 & 106592594 \\
\hline Stfa2 & -350.44 & chr11 & - & 66263201 & 66267857 \\
\hline Tmprss11d & -309.96 & $\operatorname{chr} 14$ & + & 23474012 & 23536005 \\
\hline Il1f6 & -297.95 & chr3 & + & 2482479 & 2487029 \\
\hline RGD1562305_predicted & -265.17 & chr1 & + & 85796214 & 85800682 \\
\hline RGD1311975 & -239.31 & chr9 & + & 56879058 & 56938623 \\
\hline Slpi & -179.45 & chr3 & - & 155373824 & 155376074 \\
\hline RGD1561909 & -165.00 & chr1 & + & 72833802 & 72836472 \\
\hline RGD1562060 & -158.11 & chr5 & - & 17414522 & 17438458 \\
\hline Kb1 & -152.57 & chr7 & - & 140540959 & 140546186 \\
\hline Rhcg & -149.21 & chr1 & - & 135361417 & 135386461 \\
\hline Tgm3 & -147.44 & $\operatorname{chr} 3$ & + & 117622726 & 117659103 \\
\hline Il1f8 & -138.56 & chr3 & - & 2510814 & 2515661 \\
\hline Slpil3 & -137.05 & chr3 & - & 155348325 & 155349745 \\
\hline Lce1f & -135.61 & chr2 & - & 185714947 & 185716534 \\
\hline Adh6a & -129.56 & chr2 & + & 235842524 & 235850491 \\
\hline Acpp & -125.57 & chr8 & - & 109363250 & 109400296 \\
\hline Mt4 & -119.65 & chr19 & - & 11300056 & 11302153 \\
\hline S100vp & -117.23 & chr2 & - & 183734038 & 183737388 \\
\hline Krt10 & -116.88 & $\operatorname{chr} 10$ & - & 88288619 & 88292615 \\
\hline Sprr3 & -115.55 & chr2 & - & 185436597 & 185437356 \\
\hline Klk8 & -101.32 & chr1 & + & 94227184 & 94234028 \\
\hline Krt1-12 & -100.29 & chr10 & - & 88321125 & 88328759 \\
\hline Tgm1 & -99.73 & chr15 & - & 33846366 & 33859615 \\
\hline Tnn & -98.87 & $\operatorname{chr} 13$ & - & 75502878 & 75570266 \\
\hline Defb5 & -96.76 & chr16 & - & 75403110 & 75405271 \\
\hline RGD1563060 & -85.47 & $\operatorname{chr} 18$ & - & 37217041 & 37242030 \\
\hline S100a7a & -83.60 & chr2 & + & 182946272 & 182950714 \\
\hline LOC290595 & -81.85 & $\operatorname{chr} 16$ & - & 13308804 & 13316412 \\
\hline RGD1562464 & -81.81 & $\operatorname{chr} 13$ & + & 88561387 & 88569578 \\
\hline LOC691259 & -80.74 & $\operatorname{chr} 10$ & + & 44018454 & 44029319 \\
\hline Klk10 & -75.76 & chr1 & + & 94210923 & 94214301 \\
\hline Crispld1 & -71.58 & chr5 & - & 343492 & 383772 \\
\hline MGC72615 & -70.21 & chr4 & - & 70401083 & 70419847 \\
\hline Klk11 & -66.19 & chr1 & + & 94204124 & 94208665 \\
\hline RGD1562059 & -65.57 & chr2 & + & 189315150 & 189321030 \\
\hline
\end{tabular}

absorption, steroid biosynthesis, mineral absorption, amoebiasis, rheumatoid arthritis, p53 signalling, glycine, serine and threonine metabolism, MAPK signalling, cell cycle regulation, tuberculosis (Fig. 3D). 

Cellular Physiology Cell Physiol Biochem 2015;37:1513-1526 \begin{tabular}{l|l|l|l}
\hline DOI: 10.1159/000438519 & 0215 The Author(s). Published by S. Karger AG, Basel
\end{tabular} and Biochemistry Published online: October 30, 2015 www.karger.com/cpb
Pan et al.: LncRNA Expression Profiling in A-ED Rats

Table 6. LncRNAs are differentially expressed in A-ED vs. NC, as validated by microarray analysis and RTPCR

\begin{tabular}{|c|c|c|c|c|c|c|c|}
\hline SeqID & Microarray & qPCR & Chromosome & Strand & Relationship & Associated_gene_acc & Associated_gene_name \\
\hline MRAK080737 & 16.78 & 2.956756 & chr4 & $\cdot$ & bidirection & NM_022522 & Casp2 \\
\hline MRuc008sut & 7.88 & 5.631329 & chr5 & + & sense_exon_overlap & NM_031628 & $\mathrm{Nr} 4 \mathrm{a} 3$ \\
\hline AB049626 & 3.34 & 2.488023 & chr 4 & + & sense_intron_overlap & NM_013146 & Cald1 \\
\hline MRNR_026574 & 3.12 & 2.359001 & chr1 & + & sense_exon_overlap & NM_001143847 & Pde2a \\
\hline MRAK161159 & 3.04 & 1.886605 & $\operatorname{chr} 11$ & + & sense_intron_overlap & NM_032062 & Kalrn \\
\hline MRAK040414 & 2.13 & 4.575866 & chr5 & - & sense_exon_overlap & NM_022383 & Cap1 \\
\hline MRAK153573 & 2.05 & 2.485457 & chr2 & + & sense_exon_overlap & NM_012519 & Camk2d \\
\hline MRAK078930 & -8.03 & 0.321868 & chr1 & $\cdot$ & sense_exon_overlap & NM_013159 & Ide \\
\hline BC167026 & -7.49 & 0.413323 & chr15 & + & sense_exon_overlap & NM_001106028 & Cdkn3 \\
\hline MRAK142111 & -2.99 & 0.350863 & $\operatorname{chr} 4$ & + & sense_exon_overlap & NM_053356 & Col1a2 \\
\hline BC082040 & -2.83 & 0.424388 & chr12 & + & sense_intron_overlap & NM_031338 & Camkk2 \\
\hline MRAK084895 & -2.54 & 0.268796 & chr6 & $\cdot$ & sense_exon_overlap & NM_019153 & Fbln5 \\
\hline
\end{tabular}

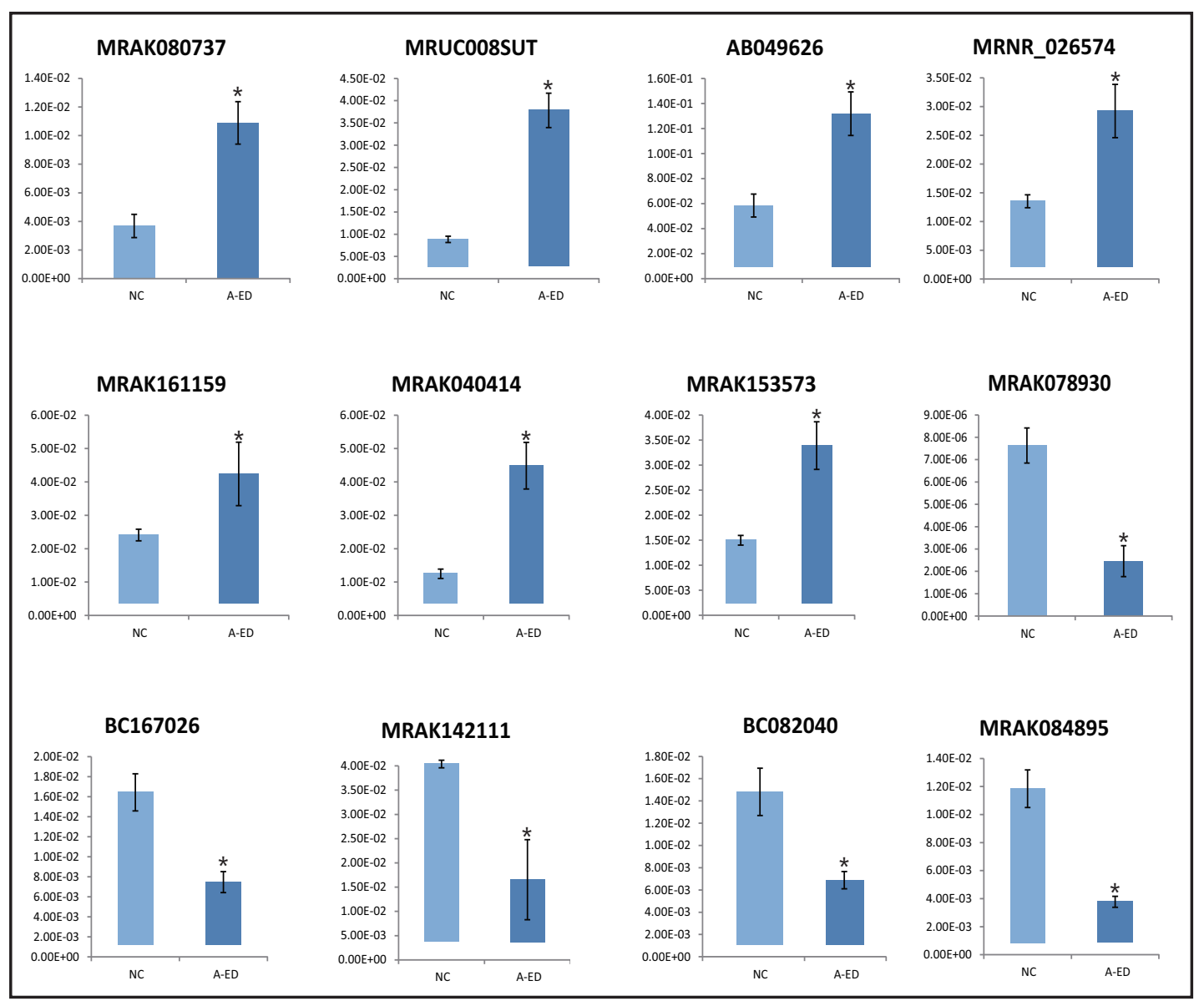

Fig. 2. The differential expression of IncRNAs between A-ED and NC tissues by quantitative PCR, ${ }^{*} p<0.05$.

\section{Discussion}

Erectile dysfunction (ED), which is defined as the inability of a man to achieve and maintain an erection sufficient for mutually satisfactory intercourse with his partner, usually causes stress and relationship problems and affects male self-confidence [25]. Little attention 


\section{Cellular Physiology Cell Physiol Biochem 2015;37:1513-1526 \begin{tabular}{l|l} 
and Biochemistry 10.1159/000438519 & ( ) 2015 The Author(s). Published by S. Karger AG, Basel
\end{tabular}

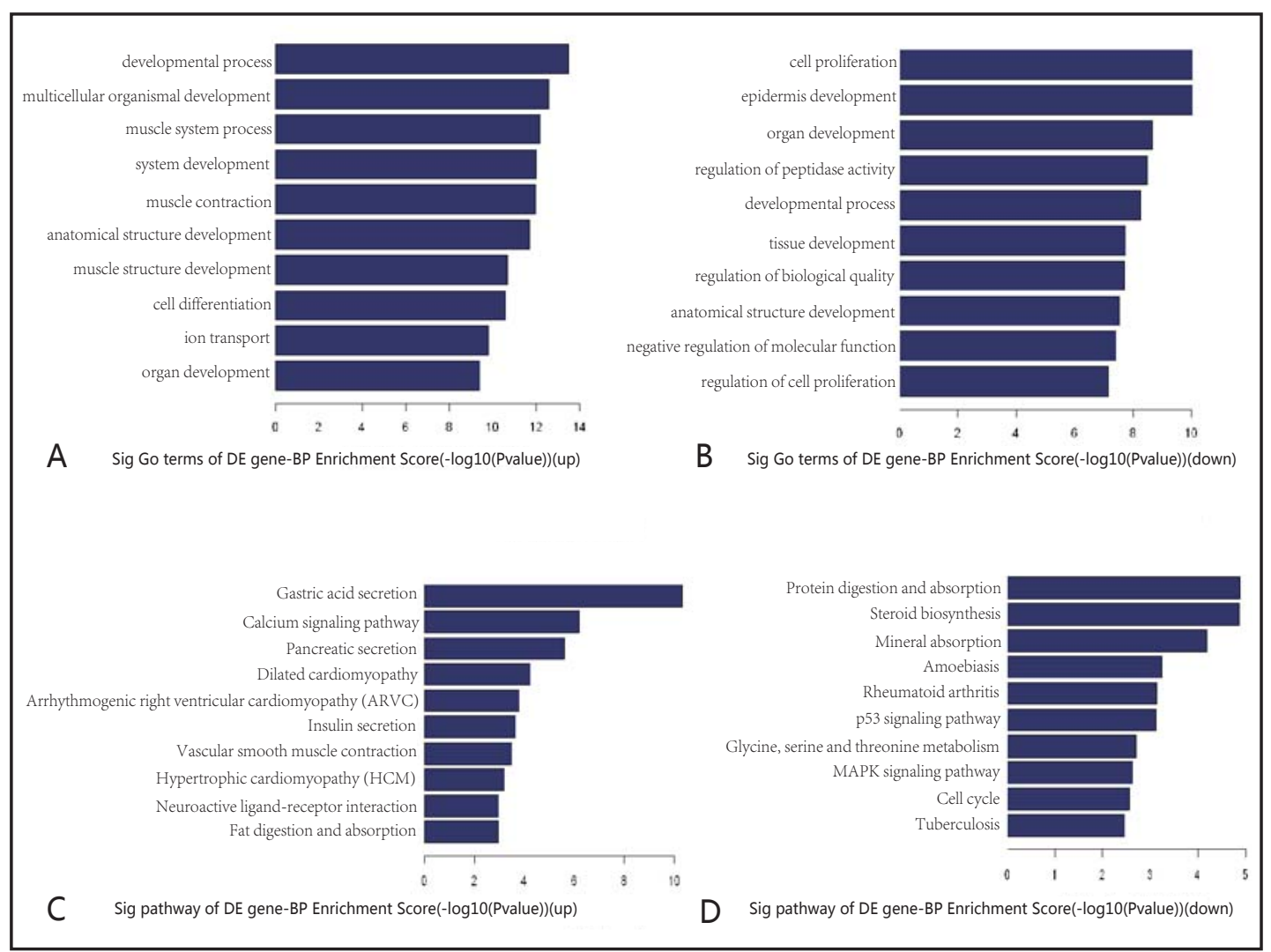

Fig. 3. GO and pathway analysis. The bar plot shows the top $10 \mathrm{GO}$ terms that are associated with the coding genes of up-regulated IncRNAs (A) and down-regulated IncRNAs (B). The bar plot shows the top 10 pathways that are associated with the coding genes of up-regulated lncRNAs (C) and down-regulated lncRNAs (D).

is given to sexual behaviour and to the treatment of ED in the older population, due to the general hypotheses that a loss of sexuality was natural and inevitable part of aging. However, in the current era, elderly men continue to be sexually active, and there is no upper age limit for sexual function $[5,26,27]$. Despite the huge success of oral phosphodiesterase-5 inhibitors (PDE5I), about one third of ED patients do not respond to PDE5I, which is especially true for older patients [1]. Therefore, ED remains a topic of interest to physicians treating aging and older males.

The genome is extremely intricate and can be transcribed into a spectrum of ncRNAs that are implicated in a wide range of structural, regulatory, and catalytic processes [2831]. Some of these ncRNAs are well known, such as short hairpin RNAs (shRNAs), small interfering RNAs (siRNAs) and microRNAs (miRNAs), and the roles of these RNAs in the progression of erectile function have been studied, at least in part [6, 32, 33]. LncRNAs are one of the most abundant groups of ncRNAs, which have been reported to have in a wide range of physiological functions. However, the roles of lncRNA in the pathogenesis of ED are poorly understood.

In this study, we attempted to explore and describe lncRNAs expression signatures of A-ED using a 2nd-generation IncRNA microarray. The lncRNA microarray analysis detected a total of 8,744 lncRNAs and 13,585 coding transcripts. However, only 608 up-regulated and 406 down-regulated lncRNAs were identified in A-ED compared with NC, as determined by setting a filter of fold-change $>2.0$. The results of the qRT-PCR analysis were consistent with the microarray results and demonstrated that the microarray data were reliable. Until now, few studies have been reported on these 12 randomly selected lncRNAs and little is 


\section{Cellular Physiology Cell Physiol Biochem 2015;37:1513-1526 \\ and Biochemistry \begin{tabular}{l|l} 
DOI: 10.1159/000438519 & $\begin{array}{l}\text { C } 2015 \text { The Author(s). Published by S. Karger AG, Basel } \\
\text { www.karger.com/cpb }\end{array}$ \\
\cline { 1 - 2 }
\end{tabular} \\ Pan et al.: LncRNA Expression Profiling in A-ED Rats}

known about the functions of these lncRNAs in physiological and pathological processes. However, based on their significantly up-regulated or down-regulated expression levels in A-ED samples, we hypothesize that some of the lncRNAs might be involved in the regulation of the progression of A-ED which should be investigated in future studies. In addition, the unique lncRNA expression signatures of these samples prompt a potential novel way to diagnose and remedy A-ED. However, it is too premature to use these regulated IncRNAs as biomarkers of A-ED. Further studies to investigate the structure and function of these lncRNAs are necessary.

GO term enrichment analysis was performed to identify the biological processes associated with the lncRNAs that we identify as differentially expressed. We found that the IncRNAs with differential expression are most highly enriched in functions related to muscle contraction, muscle system process, muscle structure development, and ion transport. Which are closely connected to penile erection. Moreover, pathway analysis showed a significant change in the pathways for calcium signalling pathway, vascular smooth muscle contraction, mineral absorption, MAPK signalling and protein digestion and absorption during A-ED. Normal erectile function is based on the coordination of activity of the corporal smooth muscle cells. Ionic gradients play an important role in the normal physiology of the corporal smooth muscle cell [34]. The result suggests that a muscle contraction disorder induced by abnormal ion channels might play a critical role in the pathogenesis of A-ED.

In previous studies, copious collagen was found in human erectile tissues, and altered collagen content was associated with erectile dysfunction [1, 35, 36]. Immunohistochemistry analysis revealed that in the corpus cavernosum, collagen types I and IV predominate, and type III is in the minority [37]. Col1a2 is one of the collagen gene families that directly participate in collagen deposition and fibrosis [38, 39]. A recent study demonstrated that lncRNAs contributed to reduced expression of collagen [40]. Research on idiopathic pulmonary fibrosis indicated that interactions between microRNAs, IncRNAs and mRNAs might better explain the disease pathogenesis [41]. Our results revealed that MRAK142111 expression was decreased, which is associated with Col1a2. Thus, MRAK142111 may be involved in the regulation of collagen deposition and fibrosis in the corpus cavernosum.

Vascular smooth muscle contraction is an important part in erectile function. The function of smooth muscle cell in the corpus cavernosum is controlled by a balance between factors for contraction and extension [1]. Berridge et al. [42] found that smooth muscle contraction is controlled by calcium signalling pathways. Camk2d is part of the calcium signalling pathways and involved in actin cytoskeleton reorganization [43]. Other studies revealed that camk2d participates in the function of vascular smooth muscle cells by regulating cell polarization, migration and proliferation [44, 45]. MRAK153573 expression was increased during A-ED progress in our study, and its associated gene was Camk2d. Thus, MRAK153573 may be very important for the functions of vascular smooth muscle and might contribute to the pathophysiology of A-ED.

The molecular mechanisms of lncRNAs are largely unknown. Unlike protein-coding genes or microRNAs, IncRNAs sequences generally do not provide direct information to predict their function [46]. LncRNAs could be implicated in gene regulation through multifarious mechanisms such as posttranscriptional gene regulation, alternative splicing and epigenetic modifications of DNA [47-49]. Moreover, lncRNAs and other molecules such as protein complex, miRNA, and other regulatory elements form a complex interaction network [50]. Accumulating associations among lncRNAs and other molecules are being verified by experimental means or are being predicted by computational methods $[51,52]$. StarBase (http://starbase.sysu.edu.cn) is designed for decoding the interaction networks of lncRNAs, microRNAs, competing endogenous RNAs (ceRNAs), RNA-binding proteins (RBPs) and mRNAs. To determine the role of ncRNAs in various diseases and pathway, the annotation of miRNA regulatory elements and targets can be obtained from DIANA-LncBase at www.microrna.gr/LncBase. miRWalk2.0 (http://zmf.umm.uni-heidelberg.de/apps/ $\mathrm{zmf} / \mathrm{mirwalk} 2 /$ ] [53] is an improved version of miRWalk [54], which supplies the biggest available collection of predicted and experimentally verified miRNA-target interactions with 


\section{Cellular Physiology Cell Physiol Biochem 2015;37:1513-1526 \begin{tabular}{ll|l} 
DOI: 10.1159/000438519 & $\begin{array}{l}\text { C } 2015 \text { The Author(s). Published by S. Karger AG, Basel } \\
\text { www.karger.com/cpb }\end{array}$
\end{tabular} \\ Pan et al.: LncRNA Expression Profiling in A-ED Rats}

various novel and unique features including lncRNAs. Further research on exploring the possibility interactions of our dataset will be important and necessary to understand the molecular biology of A-ED.

In this study, we profiled the expression of IncRNAs and mRNAs in A-ED and NC animals. Our results indicate that several IncRNAs are involved in A-ED development. Further study will be necessary to investigate the molecular mechanisms of specific dysregulated lncRNAs, which may be helpful for the exploration of novel therapeutic targets in A-ED.

\section{Conclusion}

We studied the profile of differentially expressed lncRNAs between A-ED and NC rats. The data indicates that many lncRNAs are involved in the regulation of muscle contraction and relaxation through ion channel activity. We suggest that a muscle contraction disorder induced by abnormal ion channel activity, collagen deposition and fibrosis might play a critical role in the pathogenesis of A-ED. However, our study is a preliminary exploration of the IncRNA profile in A-ED. Additional research is necessary to understand the roles of differentially expressed lncRNAs.

\section{Acknowledgements}

This study was funded by the National Natural Science Foundation of China (30972995) and was supported by the Science and Technology Development Fund of the Nanjing Medical University (2013NJMU127 and 2014NJMUZD051), the Scientific Research Project of the Health Department of Jiangsu Province (Z201022) and the Science and Technology project of Zhenjiang (SH2011042).

\section{Disclosure Statement}

We have no disclosure statement.

\section{References}

1 Andersson KE: Mechanisms of penile erection and basis for pharmacological treatment of erectile dysfunction. Pharmacol Rev 2011;63:811-859.

2 Gareri P, Castagna A, Francomano D, Cerminara G, De Fazio P: Erectile dysfunction in the elderly: an old widespread issue with novel treatment perspectives. Int J Endocrinol 2014;2014:878670.

3 Melman A, Gingell JC: The epidemiology and pathophysiology of erectile dysfunction. J Urol 1999;161:5-11.

4 Nicolosi A, Laumann EO, Glasser DB, Moreira ED Jr, Paik A, Gingell C: Sexual behavior and sexual dysfunctions after age 40: the global study of sexual attitudes and behaviors. Urology2004;64:991-997.

5 Albersen M, Orabi H, Lue TF: Evaluation and treatment of erectile dysfunction in the aging male: a minireview. Gerontology 2012;58:3-14.

6 Pan F, Xu J, Zhang Q Qiu X, Yu W, Xia J, Chen T, Pan L, Chen Y, Dai Y:Identification and Characterization of the MicroRNA Profile in Aging Rats with Erectile Dysfunction. J Sex Med 2014;11:1646-1656.

7 Kapranov P, Cheng J, Dike S, Nix DA, Duttagupta R, Willingham AT, Stadler PF, Hertel J, Hackermüller J, Hofacker IL, Bell I, Cheung E, Drenkow J, Dumais E, Patel S, Helt G, Ganesh M, Ghosh S, Piccolboni A, Sementchenko V, Tammana H, Gingeras TR: RNA maps reveal new RNA classes and a possible function for pervasive transcription. Science (New York, N.Y.) 2007;316:1484-1488.

8 Mattick JS: Long noncoding RNAs in cell and developmental biology. Semin Cell Dev Biol 2011;22:327.

9 Mattick JS: The central role of RNA in human development and cognition. FEBS letters 2011;585:16001616. 


\section{Cellular Physiology Cell Physiol Biochem 2015;37:1513-1526 \begin{tabular}{l|l|l}
\hline DOI: 10.1159/000438519 & () 2015 The Author(s). Published by S. Karger AG, Basel
\end{tabular} and Biochemistry Published online: October 30, 2015 www.karger.com/cpb}

Pan et al.: LncRNA Expression Profiling in A-ED Rats

10 Qureshi IA, Mattick JS, Mehler MF: Long non-coding RNAs in nervous system function and disease. Brain Res 2010;1338:20-35.

11 Rinn JL, Kertesz M, Wang JK, Squazzo SL, Xu X, Brugmann SA, Goodnough LH, Helms JA, Farnham PJ, Segal E, Chang HY: Functional demarcation of active and silent chromatin domains in human HOX loci by noncoding RNAs. Cell 2007;129:1311-1323.

12 Tahira AC, Kubrusly MS, Faria MF, Dazzani B, Fonseca RS, Maracaja-Coutinho V, Verjovski-Almeida S, Machado MC, Reis EM: Long noncoding intronic RNAs are differentially expressed in primary and metastatic pancreatic cancer. Mol Cancer 2011;10:141.

13 Aryal B, Rotllan N, Fernández-Hernando C: Noncoding RNAs and atherosclerosis. Curr Atheroscler Rep 2014;16:407.

14 Holdt LM :Alu elements in ANRIL non-coding RNA at chromosome 9p21 modulate atherogenic cell functions through trans-regulation of gene networks. PLoS genetics 2013;9:e1003588.

15 Liu G, Sun X, Bian J, Wu R, Guan X, Ouyang B, Huang Y,Xiao H, Luo D, Atala A, Zhang Y, Deng C: Correction ofdiabetic erectile dysfunction with adipose derived stem cellsmodified with the vascular endothelial growth factor gene in arodent diabetic model. PLoS ONE 2013;8:e72790.

16 Heaton JP, Varrin SJ, Morales A: The characterization of abio-assay oferectile function in a rat model. J Urol 1991;145:1099-1102.

17 Chen Y, Li SX, Yao LS, Wang R, Dai YT: Valsartan treatment reverses erectile dysfunction in diabetic rats. Int J Impot Res 2007;19:366-370.

18 Khatri P, Sirota M, Butte AJ: Ten years of pathway analysis: current approaches and outstanding challenges. PLoS Comput Biol 2012;8:e1002375.

19 Nordström KJ, Mirza MA, Larsson TP, Gloriam DE, Fredriksson R, Schiöth HB: Comprehensive comparisons of the current human, mouse, and rat RefSeq, Ensembl, EST, and FANTOM3 datasets: identification of new human genes with specific tissue expression profile. BiochemBiophys Res Commun 2006;348:1063-1074.

20 Pruitt KD, Tatusova T, Maglott DR: NCBI Reference Sequence (RefSeq): a curated non-redundant sequence database of genomes, transcripts and proteins. Nucleic Acids Res 2005;33:D501-504.

21 Rosenbloom KR, Armstrong J, Barber GP, Casper J, Clawson H, Diekhans M, Dreszer TR, Fujita PA, Guruvadoo L, Haeussler M, Harte RA, Heitner S, Hickey G, Hinrichs AS, Hubley R, Karolchik D, Learned K, Lee BT, Li CH, Miga KH, Nguyen N, Paten B, Raney BJ, Smit AF, Speir ML, Zweig AS, Haussler D, Kuhn RM, Kent WJ: The UCSC Genome Browser database: 2015 update. Nucleic Acids Res 2015;43:D670-681.

22 Li Y, Wang X, Li M, Pan J, Jin M, Wang J, Li X, Feng X: Long non-coding RNA expression profile in the kidneys of male, low birth weight rats exposed to maternal protein restriction at postnatal day 1 and day 10. PLoS One 2015;10: e0121587.

23 Zhang J, Cui X, Shen Y, Pang L, Zhang A, Fu Z, Chen J, Guo X, Gan W, Ji C: Distinct expression profiles of LncRNAs between brown adipose tissue and skeletal muscle. BiochemBiophys Res Commun 2014;443:1028-1034.

24 Li J, Long W, Li Q, Zhou Q, Wang Y, Wang H, Zhou B, Li J: Distinct expression profiles of lncRNAs between regressive and mature scars. Cell Physiol Biochem2015;35:663-675.

25 Corona G, Rastrelli G, Maseroli E, Forti G, Maggi M: Sexual function of the ageing male. Best Pract Res Clin Endocrinol Metab 2013;27:581-601.

26 Lindau ST, Schumm LP, Laumann EO, Levinson W, O'Muircheartaigh CA, Waite LJ: A study of sexuality and health among older adults in the United States. N Engl J Med 2007;357:762-774.

27 Smith LJ, Mulhall JP, Deveci S, Monaghan N, Reid MC: Sex after seventy: a pilot study of sexual function in older persons. J Sex Med 2007;4:1247-1253.

28 Carninci P: The transcriptional landscape of the mammalian genome. Science 2005;309:1559-1563.

29 Cheng J: Transcriptional maps of 10 human chromosomes at 5-nucleotide resolution. Science (New York, N.Y.) 2005;308:1149-1154.

30 Kapranov P, Willingham AT, Gingeras TR: Genome-wide transcription and the implications for genomic organization. Nat Rev Genet 2007;8:413-423.

31 Mattick JS, Makunin IV: Non-coding RNA. Hum Mol Genet 2006;15:R17-29.

32 Cao ZG, Zhu YP, Sun YW, Dong XC, Qi L, Xiao J, Chen H, Ren WH, Zou L: Small interfering RNA inhibits the expression of connexin43 in the human corpus cavernosum penis smooth muscle cells. Zhonghua Nan Ke Xue 2007;13:440-443. 


\section{Cellular Physiology Cell Physiol Biochem 2015;37:1513-1526 \begin{tabular}{ll|l} 
DOI: 10.1159/000438519 & $\begin{array}{l}\text { @ 2015 The Author(s). Published by S. Karger AG, Basel } \\
\text { www.karger.com/cpb }\end{array}$ \\
\hline
\end{tabular}}

Pan et al.: LncRNA Expression Profiling in A-ED Rats

33 Magee TR, Kovanecz I, Davila HH, Ferrini MG, Cantini L, Vernet D, Zuniga FI, Rajfer J, Gonzalez-Cadavid NF: Antisense and short hairpin RNA (shRNA) constructs targeting PIN (Protein Inhibitor of NOS) ameliorate aging-related erectile dysfunction in the rat. J Sex Med 2007;4:633-643.

34 Noack T, Noack P: Multiple types of ion channels in cavernous smooth muscle. World J Urol 1997;15:45-49.

35 Mulhall JP: Penile rehabilitation following radical prostatectomy. Curr Opin Urol 2008;18:613-620.

36 User HM, Hairston JH, Zelner DJ, McKenna KE, McVary KT: Penile weight and cell subtype specific changes in a post-radical prostatectomy model of erectile dysfunction. J Urol 2003;169:1175-1179.

37 Luangkhot R,Rutchik S,Agarwal V,Puglia K,Bhargava G,Melman A: Collagenalterations in the corpus cavernosum of men with sexualdysfunction. J Urol 1992;148:467-471.

38 Tamaki Z, Asano Y, Kubo M, Ihn H, Tada Y, Sugaya M, Kadono T, Sato S: Effects of the immunosuppressant rapamycin on the expression of human $\alpha 2$ (I) collagen and matrix metalloproteinase 1 genes in scleroderma dermal fibroblasts. J DermatolSci 2014;74:251-259.

39 Dong J, Porter DW, Batteli LA, Wolfarth MG, Richardson DL, Ma Q: Pathologic and molecular profiling of rapid-onset fibrosis and inflammation induced by multi-walled carbon nanotubes. Arch Toxicol 2015;89:621-633.

40 Liu Q, Zhang X, Dai L, Hu X, Zhu J, Li L, Zhou C, Ao Y: Long noncoding rna related to cartilage injurypromotes chondrocyte extracellular matrix degradation in osteoarthritis. Arthritis Rheumatol 2014;66:969-978.

41 Huang C, Yang Y, Liu L: Interaction of long non-coding RNAs and microRNAs in the pathogenesis of idiopathic pulmonary fibrosis. Physiol Genomics DOI:10.1152/physiolgenomics.00064.2015.

42 Berridge MJ: Smooth muscle cell calcium activation mechanisms. J Physiol 2008;586:5047-5061.

43 Cai H, Liu D, Garcia JG: CaM Kinase II-dependentpathophysiological signalling in endothelial cells. Cardiovasc Res2008;77:30-34.

44 Mercure MZ, Ginnan R, Singer HA:CaM kinase II delta2-dependent regulation of vascular smooth muscle cell polarization and migration. Am J Physiol Cell Physiol 2008;294:C1465-1475.

45 House SJ, Ginnan RG, Armstrong SE, Singer HA: Calcium/calmodulin-dependent protein kinase II-delta isoform regulation of vascular smooth muscle cell proliferation. Am J Physiol Cell Physiol 2007;292:C22762287.

46 Guo X, Gao L, Wang Y, Chiu DK, Wang T, Deng Y: Advances in long noncoding RNAs: identification, structure prediction and function annotation. Brief Funct Genomics 2015;pii:elv022.

47 Mercer TR, Mattick JS: Structure and function of long noncodingRNAs in epigenetic regulation. Nat Struct Mol Biol 2013;20:300-307.

48 Tripathi V1, Ellis JD, Shen Z, Song DY, Pan Q, Watt AT, Freier SM, Bennett CF, Sharma A, Bubulya PA, Blencowe BJ, Prasanth SG, Prasanth KV: The nuclear-retained noncodingRNA MALAT1 regulates alternative splicing by modulatingSR splicing factor phosphorylation. Mol Cell 2010;39:925-938.

49 Yoon JH1, Abdelmohsen K, Srikantan S, Yang X, Martindale JL, De S, HuarteM, Zhan M, Becker KG, Gorospe M: LincRNAp21suppresses target mRNA translation. Mol Cell 2012;47:648-655.

50 Guttman M, Rinn JL. Modular regulatory principles of large non-coding RNAs. Nature 2012;482:339-246.

51 Chu C, Qu K, Zhong FL, Artandi SE, Chang HY: Genomic maps of long noncodingRNA occupancy reveal principles of RNA-chromatininteractions. Mol Cell 2011;44:667-678.

52 Wang Y, Chen X, Liu ZP, Huang Q, Wang Y, Xu D, Zhang XS, Chen R, Chen L: De novo prediction of RNAprotein interactions from sequence information. Mol Biosyst 2013;9:133-142.

53 Dweep H, Gretz N: miRWalk2.0: a comprehensive atlas of microRNA-target interactions. Nat Methods 2015;12:697.

54 Dweep H, Sticht C, Pandey P, Gretz N: miRWalk--database: prediction of possible miRNA binding sites by "walking" the genes of three genomes. J Biomed Inform 2011;44:839-847. 